\title{
Poly C binding Protein 1 Represses Autophagy through Downregulation of LC3B to Promote Tumor Cell Apoptosis in Starvation
}

Wenliang Zhang ${ }^{1,2,6}$, Hongshun Shi ${ }^{1,6}$, Mingming Zhang ${ }^{1}$, Bin Liu ${ }^{1}$, Shuai Mao ${ }^{3} \mathrm{Li} \mathrm{Li}^{1}$, Fang Tong ${ }^{1}$, Guoliang Liu ${ }^{5}$, Shulan Yang ${ }^{2 *}$, Haihe Wang ${ }^{1,4^{*}}$

${ }^{1}$ Department of Biochemistry, Zhongshan School of Medicine

${ }^{2}$ Translational Medicine Centre, the First Affiliated Hospital

${ }^{3}$ Department of Hepatic Surgery, the First Affiliated Hospital

${ }^{4}$ Center for Stem Cell Biology and Tissue Engineering.

Sun Yat-sen University, Guangzhou, CPZN 510080, People's Republic of China

${ }^{5}$ Department of Internal Medicine, Jinxian People's Hospital of Nanchang, Nanchang, CPZN 331700, China

${ }^{6}$ Co-first authors

*Correspondences: Shulan Yang, No. 58 Zhonshan Road 2 $2^{\text {nd }}$ E-mail: yangsh13@mail.sysu.edu.cn or Haihe Wang, No. 74, Zhonshan Road 2 ${ }^{\text {nd }}$, Guangzhou, CPZN 510080, People's Republic of China, Telephone: (86)-20-87335035, E-mail: wanghaih@mail.sysu.edu.cn.

Running Title: PCBP1 represses autophagy to promote apoptosis

Keywords: PCBP1; Autophagy; Apoptosis; LC3B; Cancer

Conflict of interest: The authors declare no conflicting interests 


\section{Abstract}

Accumulating evidences indicate that Poly C Binding Protein (PCBP1) is downregulated in various carcinomas as a tumor suppressor, but the underlying mechanism in suppression of tumorigenesis still remains elusive. Here, we found that PCBP1 overexpression attenuates tumor cell growth upon serum-free starvation. Notably, the autophagic degradation inhibitor, chloroquine, could mimick this suppressive effect in tumor cell growth. Autophagy analyses demonstrated that PCBP1 overexpression blocked autophagic flux of tumor cells under starvation conditions, while PCBP1 downregulation in turn refueled this autophagic flux, protecting cells from death. Mechanistically, PCBP1 overexpression attenuated microtubule-associated protein Light chain 3 (LC3B) mRNA stability to repress LC3B expression, resulting in the autophagy inhibition. Consequently, PCBP1 overexpression strongly triggered the caspase 3 and 8-mediated apoptosis of tumor cells and downregulated anti-apoptotic Bcl-2 expression upon starvation, which could be further synergized by autophagic inhibitor, indicating that PCBP1 not only inhibits tumor cell autophagy, but also renders them to apoptosis. Taken together, our results uncovered a novel mechanism of PCBP1 in repressing autophagy-mediated cell survival and indicated that inhibition of tumor cell autophagy by PCBP1 upregulation or with autophagic inhibitors could be an effective therapeutical strategy to colon and ovary tumors with low PCBP1 expression.

\section{Introduction}

Autophagy is a conserved catabolic pathway hallmarked by the double-membrane 
vesicles formation (autophagosomes) that can swallow cytoplasmic organelles and proteins, and then conjugate to lysosomes for their luminal content degradation. Autophagy putatively functions as a cytoprotective manner to favor stress adaptation and to prevent cell death. In specific conditions, it may constitute an alternative pathway to cellular demise (autophagic cell death) (Maiuri et al. , 2010, Mizushima, 2007). Starvation, rapamycin, hypoxia and other stresses can induce autophagy. During autophagy, the mammalian homolog of yeast Atg8, microtubule-associated protein Light Chain 3 (LC3), undergoes turnover from LC3 I to LC3 II and serves as a reliable marker of autophagosomes (Klionsky et al. , 2012). The p62/ SQSTM1 (p62) protein, serving as a link between LC3 and ubiquitinated substrates, is incorporated into the completed autophagosome and is also significantly degraded in autolysosomes, when autophagy is induced (Bjorkoy et al. , 2005, Pankiv et al. , 2007). Autophagic flux can be measured by inferring LC3-II and p62 degradation with western blotting and immuostaining (Klionsky, Abdalla, 2012). The current consensus show that autophagy have dual roles in the development of tumors (Galluzzi et al. , 2015, Levine, 2007, Mizushima, 2007). Generally, cells in the center of tumors are deprived of nutrition and have higher autophagic flux than cells in the margins of tumors to prevent their death (Degenhardt et al. , 2006). On the other hand, autophagy also exists in dying cells, and can render the eventual cell death via the excessive consumption of cellular portions (Huang et al. , 2013, Janku et al. , 2011, Young et al. , 2012). Thus, roles of autophagy in cancer progression are highly dependent on physiological and pathological conditions. So far, it still remains elusive that which factor modulates starvation-induced autophagy and determines the eventual destiny of 
tumor cells.

Apoptosis is well characterized by the activation of caspases, putatively known as a family of cysteine proteases (Fulda and Debatin, 2006, Li and Yuan, 2008). Caspase-8 is an initiator caspase whose auto-activation starts from its oligomerization and self-cleavage, and the activated caspase- 8 subsequently activates executioner caspases, such as pro-caspase-3, to facilitate the apoptotic cleavage of poly (ADP-ribose) polymerase (PARP) for the programmed cell death (Kruidering and Evan, 2000). Recent evidences suggested that crosstalking between autophagy and apoptosis can coordinately regulate cell death (Wu et al. , 2014).

Poly $\mathrm{C}$ binding protein 1 (PCBP1) is a well known RNA binding protein and participates in multiple gene regulatory processes, including transcriptional activation, RNA splicing, translation enhancement or silence, and transportation process via its poly (C)-binding ability (Giles et al. , 2003, Malik et al. , 2006, Nishinakamura et al. , 2007, Rivera-Gines et al. , 2006, Wang et al. , 2010, Zhang et al. , 2010). Previously, we reported that PCBP1 suppresses the translation of metastasis-associated PRL-3 phosphatase, and showed that PCBP1 is widely downregulated in various tumors, including colon, and lung carcinomas, indicating PCBP1 could be a novel tumor suppressor (Wang, Vardy, 2010). In line with a recent study that PRL-3 activated autophagy and promoted cell proliferation under serum or serum-free conditions (Huang et al. , 2014), we found that PCBP1, as the suppressor of PRL-3, inhibited the growths of ovarian and colorectal tumor cells, and sought to investigate whether PCBP1 is directly linked to starvation-induced autophagy to delay tumorigenesis. Here, we reported that PCBP1 not only represses starvation-induced 
autophagy, but also enhances tumor cell apoptosis. Collectively, our results uncovered a novel evidence of PCBP1 in autophagy suppression and apoptosis enhancement during ovarian and colon tumor progression, and dislose the underlying mechanism of PCBP1 depletion to tumor formation. Thus, the expression status of PCBP1 could be a predictive biomarker, and anti-autophagic administration would be an alternative therapeutical strategy for patients with low level of PCBP1 expression and high autophagy level.

\section{Materials and Methods}

\subsection{Cell culture and treatment}

Human colorectal carcinoma cell lines DLD-1 and HCT-116, human ovarian carcinoma cell line A2780 and Chinse hamster ovary cell, CHO were purchased from ATCC and maintained in DMEM basic medium (Gbico) with 10\% FBS (invitrogen) and $1 \%$ antibiotic. All cells were incubated in $5 \% \mathrm{CO}_{2}$ at $37{ }^{\circ} \mathrm{C}$. For Earle's balanced salts solution (EBSS, Gbico) starvation treatment, cells were washed with EBSS twice and then maintained in EBSS for the indicated time. Unless otherwise specified, reagents were used at the following final concentrations: Chloroquine diphosphate salts (CQ, Sigma-Aldrich) were dissolved in PBS at concentration of $50 \mu \mathrm{M}$ and directly added to cell culture medium, and equal volume of 1x PBS alone was used as a negative control.

\subsection{DNA Constructs and Transfection}

The A2780-PCBP1, DLD-1-PCBP1 stable cells were established as previously described (Wang, Vardy, 2010). Four specific shRNA constructs of PCBP1 were bought from OriGene Technologies (USA). Transfections of tumor cells with the indicated 
protein-encoding plasmids or shRNAs were carried out with Lipofectamine 2000 (Invitrogen) as the supplier's instructions.

\subsection{Antibodies and Western blots (WB)}

Antibodies against LC3B (Cat No. 3868, WB 1:1000, IF 1:200), p62/SQSTM 1 (Cat No. 5114, WB 1:1000), Caspase-3 (Cat No. 9662, WB 1:1000), c-caspase-3(Cat No. 9661, WB 1:1000), PARP (Cat No. 9542, WB 1:1000), c-PARP (Cat No. 9541, WB 1:1000) and Bcl-2 (Cat No. 2870, WB 1:1000), Anti-rabbit HRP-labeled secondary antibody (Cat No. 7074, WB 1:2000), anti-mouse HRP-labeled secondary antibody (Cat No. 7076S, WB 1:2000) were purchased from Cell Signaling Technology and used at the indicated dilution for western blots (WB) and immunofluorescence (IF). Antibodies against Caspase-8 (Cat No.13423-1-AP, WB 1:500, Protein Tech, China), PCBP1 (Cat No. sc-137249, WB 1:500, Santa Cruz) and GAPDH (Cat No. CW0101A, WB 1:2000, CW Bio-tech, China) were also used.

For western blots analyses, cells were lysed with lysing buffer (10 mM Tris-HCl, pH 7.4; $150 \mathrm{mM} \mathrm{NaCl} ; 0.5 \mathrm{NP}-40 \% ; 1 \mathrm{mM}$ EDTA; $0.2 \mathrm{mM}$ PMSF and protease inhibitor cocktail). Whole cell lysates were centrifuged at $14,000 \mathrm{rpm}$ for $10 \mathrm{~min}$ at $4{ }^{\circ} \mathrm{C}$, then the supernatant was collected and diluted by 5x loading buffer containing 5\% SDS, 5\% 2-Mercaptoethanol and separated via SDS-polyacrylamide gel electrophoresis (SDS-PAGE) and transferred to Nitrocellulose membrane (Millipore), followed by incubation with the indicated antibodies and visualized by chemiluminescent substrates (Millipore) and exposed on radiography films. Protein band intensity was quantified and analyzed with densitometry and Image $\mathbf{J}$ software. 


\subsection{Immunofluorescence Microscopy}

Cells seeded on cover slips were fixed with $4 \%$ paraformaldehyde (PFA) in 1x PBS for 15 min, and permeabilized with $0.2 \%$ Triton X-100 (BIOSHARP) for another $30 \mathrm{~min}$ at room temperature. Cells were then blocked with 5\% BSA in PBS for $1 \mathrm{hr}$ at room temperature and incubated with primary antibodies overnight at $4{ }^{\circ} \mathrm{C}$. After washing 5 times with $1 \mathrm{x}$ PBS, cells were stained with Alexa-594-conjugated secondary antibody at room temperature for $1 \mathrm{~h}$. Cells were then washed with $1 \mathrm{x}$ PBS and incubated with DAPI for 5 min and mounted with Anti-fade Reagent and observed under LSM710 confocal microscope (Carl Zeiss AG).

\subsection{Autophagy Assays}

Autophagic flux was tested based on rate of LC3B II turnover and amount of endogenous LC3B puncta, and validated with p62 protein level for autopahgy completion. For LC3B II turnover, cells were pre-treated with $50 \mu \mathrm{M}$ chloroquine (CQ) combined with EBSS starvation, or serum deprivation at indicated time, followed by harvesting for western blotting analysis of LC3B II protein level. For endogenous LC3B staining, cells were pre-treated with $50 \mu \mathrm{M}$ CQ combined EBSS starvation for $4 \mathrm{~h}$ and then fixed for Immunofluorescence with LC3B antibody. The average LC3B channel pixel intensity value within the cytoplasmic area per cell was determined by Photoshop (Adobe Systems) to represent the overall amount of LC3B puncta accumulation in each cell. The puncta numbers were presented as mean $\pm \mathrm{SD}$.

\subsection{Cell Counting Kit-8 (CCK8) Assay}

Cells were seeded into 96-well plates in triplicate (5000 cells per well). Cells were cultured 
overnight, and on the next day, cultured medium was replaced with $100 \mu$ serum-free media with or without CQ at indicated time. CCK8 reagent (BB-4202-1, Best Bio, China) was added in each well $(10 \mu \mathrm{l}$ per well $)$. Cells were incubated with CCK8 reagent at $37^{\circ} \mathrm{C}$ for $2 \mathrm{~h}$ and the absorbance at $450 \mathrm{~nm}$ was measured by enzyme-labeling instrument (GEN10S-BASIC, Tecan Austria GmbH 5052 Grodig, Austria)

\subsection{Reverse transcriptase-polymerase chain reaction (RT-PCR)}

RT-PCR was performed by reverse transcription of $5 \mu \mathrm{g}$ total RNA and subsequent polymerase chain reaction using M-MLV Reverse Transcriptase (Promega, M1705). Primers of 5'-TACAAGGGAAGTGGCTATC-3' (Forward) and 5'-TTACACTGACAATTTCATCC-3' (Reverse) were used for RT-PCR amplification of LC3B; primers of 5'-CGGAGTCAACGGATTTGGTCGTAT-3' (Forward) and 5'-AGCCTTCTCCATGGTGGTGAAGAC-3' (Reverse) for GAPDH (Kim et al. , 2014). GoTaq ${ }^{\circledR}$ DNA polymerase (Promega, Cat No. M3005) was used in the amplification system, according to the manufacturer's protocol. In brief, the thermocycling conditions were $5 \mathrm{~min}$ at $95{ }^{\circ} \mathrm{C}$ as the first denaturation step, followed by 26 cycles at $95{ }^{\circ} \mathrm{C}$ for $30 \mathrm{~s}$, $54{ }^{\circ} \mathrm{C}$ for $30 \mathrm{~s}$ and $72{ }^{\circ} \mathrm{C}$ for $45 \mathrm{~s}$, and then extended at $72{ }^{\circ} \mathrm{C}$ for $5 \mathrm{~min}$. For normalization, expression of GAPDH was measured. DNA band intensity was quantified and analyzed with densitometry and analyzed with Image $\mathbf{J}$ software.

\subsection{Flow cytometric analysis of apoptosis}

Cells were seeded in plates $(3.5 \mathrm{~cm}$ in diameter) and cultured overnight. Next day, the culture medium was replaced with EBSS media with or without CQ for the indicated periods. Cells were detached with $0.25 \%$ EDTA-free trypsin, washed twice with 1x PBS, 
centrifuged at $2500 \mathrm{rpm}$ for $3 \mathrm{~min}$ and re-suspended in binding buffer with APC-conjugated Annexin V and 7-ADD (Cat No. KGA1025, KeyGEN BioTECH, China) at room temperature for $15 \mathrm{~min}$ in dark. The percentage of apoptotic cells was analyzed by flow cytometry (Gallios, Beckman, USA)

\subsection{Statistical analysis}

All the statistical analyses were performed by GraphPad Prism software (Version 5.00) for Windows (GraphPad Software, San Diego). Data was shown as mean + standard deviation (SD). Error bars represent the SD between the data collected from at least three independent experiments. Statistical significance was determined by Student $\mathrm{t}$ test. $P$ value $<0.05$ was considered as significant in all tests.

\section{Results}

\subsection{PCBP1 overexpression represses cancer cells proliferation under serum-free} condition.

We previously showed that PCBP1 are broadly down-regulated in various tumors, including colon, and lung carcinomas (Wang, Vardy, 2010), and recently observed that PCBP1 repressed cell proliferation, and tumorigenicity in vitro and vivo (unpublished data) under nutrition-efficient conditions. As tumor cells naturally live in nutrition-deficient and hypoxic environment, we sought to investigate whether PCBP1 could suppress tumor cell growth under this situation. We first compared the cell proliferation of A2780 and DLD-1 cells transfected with GFP control to those with GFP-PCBP1 overexpression (Figure. 1A) under serum-free starvation. CCK8 assays demonstrated that GFP-PCBP1 overexpression 
significantly inhibited growth of both A2780 and DLD-1 cells under both serum-rich and serum-free starvation conditions (Figure. 1 B and C, $P<0.001$ ). Interestingly, the autophagic inhibitor, choroquine $(\mathrm{CQ})$, simulated the effect of PCBP1 to further retard the cell growth of both A2780 and DLD-1 parental cells, leading to no statistical difference from the GFP-PCBP1 overexpression cells under this starvation (Figure. 1 B and C). Given that autophagy, as a cellular recycling mechanism, serves as a protective function to establish tumors under poorly vascularized conditions (Galluzzi, Pietrocola, 2015), we suspected that the cell proliferation inhibition by PCBP1 overexpression is due to autophagic degradation blockade, resulting in cells short of self-supplied nutrition under starvation state. To directly clarify if autophagy is really involved in this process, we checked the LC3B turnover of type I to II and found that PCBP1 clearly inhibited expression of LC3B in A2780 cells and in DLD-1 cells, upon EBBS medium-mediated starvation (Figure 1D), indicating that PCBP1 can inhibit tumor cell autophagy in starvation conditions to inhibit cell growth.

\subsection{PCBP1 overexpression represses starvation-induced autophagic flux}

To thoroughly examine how PCBP1 is involved in starvation-induced autophagy, we utilized CQ to inhibit autophagic degradation of LC3II, a hallmark of autophagic degradation. Upon EBSS-induced starvation combined with CQ treatment, we noted that PCBP1 endowed tumor cells with higher autophagic fluxes, showing with more LC3B II, compared to their parental cells, which were quantitatively shown by the ratio of LC3B II/GAPDH (Figure 2A, red boxes, lane 5 vs. lane 6 and lane 11 vs. lane 12, and Figure S1). Given autophagy is a dynamically complicated process, the elevated levels of LC3-II just 
reflect the initiation of the aurophagic process, but not necessarily for its completion, since LC3-II on the internal surface of autophagosomes can be also degraded by lysosomal enzymes of autolysosomes (Ravikumar et al. , 2010). Thus we used another late stage autophagic marker, p62 as substrate of autophagy to validate the influence of PCBP1 to autophagy. Our results revealed that p62 expression was really increased in cells with PCBP1 overexpression under normal condition (Figure 2A); but when treated with CQ, LC3B and p62 levels were not completely recovered to its GFP control level, indicating the possible negative regulation of $\mathrm{LC} 3 \mathrm{~B}$ by $\mathrm{PCBP} 1$, resulting in autophagy inhibition. Consistent with our western blotting data, immuofluorescence staining also revealed that PCBP1 overexpression obviously decreased the endogenous LC3B puncta amounts in both A2780 (Figure 2B) and DLD-1 (Figure 2C) cells upon starvation and CQ-induced autophagy inhibition, compared with GFP control cells. Quantification of LC3B puncta intensity also confirmed a dramatically lower LC3B puncta intensity per cell in PCBP1 overexpressing cells compared to the control cells, respectively (Figure 2D). Collectively, our results clearly demonstrated that PCBP1 overexpression somehow attenuated the starvation-induced autophagy in cancer cells.

\subsection{PCBP1 depletion promotes starvation-induced autophagic flux}

To verify whether the endogenous PCBP1 really participates in autophagy as the exogenous one, we next constructed PCBP1-specific shRNAs and confirmed their silencing efficiency of PCBP1 in the knockdown (KD) cells (Figure 3A). In line with our earlier observations, when inhibition of autophagy by CQ treatment, PCBP1 depletion robustly enhanced the converted LC3B-II accumulation that is indicated as ratio of 
LC3B-II to GAPDH in A2780 cells (Figure 3B, red boxes, lane 5 vs. 6) and HCT-116 cells (Figure 3B, red boxes, lane 11 vs. 12) in EBSS or serum-free starvation, compared to the parental control cells. Immunoblots results of p62 level alteration also manifested the same effects in enhancement of autophagy, when knockdown of the endogenous PCBP1 (Figure 3B). Likewise, immnofluorescent staining manifested that deletion of endogenous PCBP1 in cells (green cells) caused more endogenous LC3B puncta per cell than those in parental control cells (non-green cells) of CHO cells (Figure 3C) and HCT-116 cells (Figure 3D) under the similar combined treatments of EBSS with CQ. Thus our results showed that endogenous PCBP1 depletion reversed the starvation-induced autophagy occurrence. Together, we concluded that PCBP1 represses the degradation of starvation-induced autophagy, resulting in the cell growth inhibition in nutrient-deficient environment.

\subsection{PCBP1 overexpression downregulates LC3B expression through decreasing its}

\section{mRNA stability}

Considering that LC3B protein level can be downregulated by PCBP1 overexpression in the normal and starvation conditions, there is a possibility that PCBP1 may affect LC3B expression. Given that PCBP1 is involved in multiple gene regulatory processes as a RNA-binding protein, including modulation of mRNA stability (Giles, Daly, 2003, Malik, Flock, 2006, Nishinakamura, Minoda, 2007, Rivera-Gines, Cook, 2006, Wang, Vardy, 2010, Zhang, Huang, 2010), we sought to clarify if PCBP1 really affect LC3B expression by overexpression of GFP-tagged PCBP1 in both A2780 and DLD-1 cells or knocking down endogenous PCBP1 in A2780 cells (Figure 4A). Semi-quantitative RT-PCR results showed 
that overexpression of PCBP1 resulted in less LC3B mRNA amount, whereas knockdown of endogenous PCBP1 led to more LC3B mRNA, compared to the control cells (Figure 4B, lane 1 vs. 2 vs. 3, Figure S2). To further investigate if LC3B mRNA amount change is due to transcriptional inhibition or its mRNA stability, we first impeded the novel mRNA synthesis with Actinomycin D and check the stability of the already synthesized LC3B mRNA. Our results clearly indicated that PCBP1 somehow attenuated the synthesized LC3B mRNA stability with time course treatment, whereas, knockdown of endogenous PCBP1 counteracted this effect in A2780 cells (Figure 4B and C). The attenuated LC3B mRNA stability was also observed in DLD-1 cells with overexpression of PCBP1 (Figure 4D and E). All downregulation extents of LC3B protein levels in both A2780 and DLD-1 cells were consistent to the LC3B mRNA level changes (Figure 4F). Collectively, our results demonstrated that PCBP1 modulates LC3B expression by affecting its mRNA stability, which subsequently leads to autophagy inhibition upon starvation.

\subsection{PCBP1 overexpression enhance tumor cell apoptosis under nutrient deprivation}

As autophagy plays paradoxical roles in different stages of tumorigenesis (Sun et al. , 2013), to consequently examine the tumor cell destination with autophagy inhibition by PCBP1 in the nutrition-depleted environment, we cultured same numbers of GFP-PCBP1 expressing A2780 and DLD-1 cells and their GFP controls in EBSS, an amino acid-free medium for $36 \mathrm{~h}$. Results showed that PCBP1 overexpression induced more cell death in both A2780 (Figure 5A, a vs. a') and DLD-1 cells (Figure 5A, b vs. b'), than that of their parental cells under this nutrient-deficient environment. In addition, when further inhibition of autophagy by CQ, all GFP control cells underwent cell death as A2780 
GFP-PCBP1 cells upon EBSS starvation for 36h (Figure 5A a’ vs. c' ). Similar results were also observed in DLD-1 cells (Figure 5A, b' vs. d' ), suggesting inhibition of autophagy by CQ really promoted cell death, since CQ treatment surpassed PCBP1 suppressive effect in autophagy to render all PCBP1-expressing cell death. Quantification analyses also confirmed significantly more intensive cell death caused by PCBP1 in EBBS starvation, or in the combined CQ treatment (Figure 5B), implying that inhibition of autophagy would be a synergic strategy to eradicate tumor cells in the nutrition-deficient situation that is a common scenario in tumor environment.

Moreover, we conducted flow cytometric analyses and noticed that PCBP1 induced more percentage of apoptotic cells than those of A2780 GFP control cells under EBBS starvation alone for $24 \mathrm{~h}$, or combination with CQ treatment for $18 \mathrm{~h}(5.17 \%$ vs. $9.03 \%$; $24.31 \%$ vs. $23.33 \%)$, even in the nutrition efficient situation (3.63\% vs. $1.23 \%)$ as shown in Figure 5C. Similarly, PCBP1 led to more cells to apoptosis in DLD-1 cells under either EBSS starvation or CQ combined treatments (Figure. 5D, $7.44 \%$ vs. $2.81 \%$; $10.21 \%$ vs. $8.26 \%$ ), even in the normal culture condition (Figure 5D, $4.23 \%$ vs. 0.95\%). Taken together, our data demonstrated that PCBP1 overexpression can effectively retard tumor cell autophagy to promote tumor cell apoptosis, and combination with autophagic inhibitor can synergize cell death under starvation conditions.

\subsection{PCBP1 overexpression enhances caspase-dependent apoptosis}

To further dissect the underlying mechanism of PCBP1 in cell apoptosis upon nutrient deprivation, western blotting analyses were adopted to measure the expression and status of apoptotic proteins. In line with our above results, we noticed that the levels of 
cleaved-caspase-8, cleaved-caspase-3, and cleaved-PARP were all activated by PCBP1 overexpression in A2780 and DLD-1 cells along with starvation time, compared to that in their control cells under both normal and EBSS starvation conditions (Figure 6A and and Figure S3), indicating that PCBP1 could instinctively enhance cell apoptosis. In contrast, the anti-apoptotic protein Bcl-2 was dramatically downregulated by PCBP1 in both A2780 and DLD-1 cells, even in the normal culture situation (Figure 6A Lanes 1, 2 and Figure S3). Consistent with early observations, the apoptotic signals were further enhanced in both PCBP1 overexpressing and parental cells when autophagy degradation was blocked by CQ treatment under nutrient depletion (Figure 6A, lanes 7, 8; lanes 13, 14 and Figure S3). Similar results were also observed in HCT-116 cells when knockdown of endogenous PCBP1 in HCT-116 cells (Figure 6B). Together, our results clearly uncovered that PCBP1 overexpression combined with autophagic degradation can effectively enhance cell death under nutrient deprivation via the caspase-dependent apoptotic pathway.

\section{Discussion}

Clinical report reveals that the higher autophagy level is well correlated with the worse prognosis of ovarian carcinoma (Zhao et al. , 2014). In contrast, other evidences in turn indicate that high autophagy flux favors overall survival of ovary cancer, that is, high autophagy results in less aggressive behavior of these tumor cells with higher sensitivity to chemotherapy (Valente et al. , 2014). Our results here clarified the above dilemma and manifested that autophagy induced by nutrition depletion indeed promoted tumor cell survival. 
Currently, it is gradually understood that roles of autophagy in tumor development are highly dependent on the stage of tumor progression, physiological and pathological contexts (Galluzzi, Pietrocola, 2015, Levine, 2007, Mizushima, 2007), in which nutrition stress should be included. In early stages of tumor progression, autophagy serves as a cell survival mechanism to efficiently utilize the restricted energy when tumors' vascular supply is significantly limited (Degenhardt, Mathew, 2006, Deretic, 2008, Levine, 2007), and disruption this autophagy would definitely caused tumor cell death. Our results here clearly underlie this perspective that inhibition of autophagy in the malignant tumor cells with PCBP1 or autophagic inhibitors can efficiently sensitize these tumor cells undergo apoptosis.

Mechanistically, it is shown that ART1 gene can promote starvation-induced autophagy in colorectal CT-26 cancer cells to facilitate cell survival under shortage of nutrients (Tang et al. , 2015). However, factors regulating the starvation-induced autophagy are still rare and the underlying mechanisms remain elusive. PCBP1 is demonstrated to be widely downregulated in various tumors as a key tumor suppressor in cancer progression (Chen et al. , 2015, Liu et al. , 2015, Wang, Vardy, 2010, Zhang, Huang, 2010). We previously reported that PCBP1 downregulates metastatic phosphatase PRL-3 translation to represses tumor formation (Wang, Vardy, 2010). Recently, PRL-3 was characterized as an autophagy-driven player to promote the autophagosome nucleation and elongation or closure in A2780, CHO, DLD-1 and HCT-116 cells (Huang, Al-Aidaroos, 2014), suggesting that PCBP1 could suppress autophagy of tumor cells. Our results here clearly confirmed that PCBP1 really represses autophagic levels in starvation-induced cells, that is, 
under starvation and autophagy inhibition, PCBP1 depletion induces more autophagy flux and PCBP1overexpression in turn represses starvation-induced autophagy, implying a novel suppressive mechanism of PCBP1 in tumor formation by blocking autophagic flux to promote apoptosis under starvation situation. Furthermore, we also showed PCBP1 represses autophagy through negative modulation of LC3 expression by weakening its mRNA stability. The detailed molecular mechanism of whether PCBP1 directly participates in LC3 mRNA stability to inhibit autophagy or through a PRL-3-dependent ways will be further addressed.

Considering that starvation-induced autophagy is a cytoprotective mechanism for the development of tumors under poorly vascularized conditions, and some autophagy inhibitors, including 3-MA and CQ, are proved to enhance ovarian and colorectal tumor cell apoptosis and lead to repression of proliferation under starvation conditions (Huang, Al-Aidaroos, 2014, Lu et al. , 2014, Tang, Li, 2015), our results also evidently demonstrated that PCBP1 as a suppressor not only inhibits the starvation-induced autophagy, but can promote apoptotic cell death under this situation, implying that upregulation of PCBP1 by virus-mediated transfection could be an alternative therapeutic strategy, and inhibition of autophagy in these types of tumors can synergize the chemotherapy efficiency.

Although LC3 is the most widely used protein maker of autophagosomes in mammalian cells, even if an increase in LC3-II is observed, it is still need to determine whether this increase indicates the enhancement of autophagy flux or a block of autophagy-mediated degradation through the use of inhibitors such as chloroquine (Klionsky, Abdalla, 2012). 
p62/SQSTM1, as a substrate of autophagy, can also be monitored in this scenario. Nonetheless, as p62 contains domains that interact with several signaling molecules to participate in proteasomal degradation (Bardag-Gorce et al. , 2005, Moscat and Diaz-Meco, 2009), degradation of p62 cannot definitely assist in assessing the autophagy impairment or autophagy flux, and the combination of p62 level with LC3-II turnover should be considered together to monitor autophagy flux. Our results of decrease in p62 upon CQ treatment in a short time may also be arisen from these unknown manners, which would be further addressed, but the overall increases of LC3B level (Figure 2A) confirmed the inhibitory efficiency of CQ treatment.

In conclusion, we provided a new mechanism of PCBP1 in inhibition of tumor cell autophagy under nutrient deprivation, and blocking autophagy to apoptosis could be a potential therapeutical strategy in tumors with PCBP1 depletion.

\section{Acknowledgments}

We thank our group members for the critical comments on this manuscript. This work was supported by National Science Foundation of China (Grant No. 31271481) to WH and Guangzhou Science Technology and Innovation Commission (No. 201510010144) to YS.

\section{References}

Bardag-Gorce F, Francis T, Nan L, Li J, He Lue Y, French BA, et al. Modifications in P62 occur due to proteasome inhibition in alcoholic liver disease. Life sciences. 2005;77:2594-602.

Bjorkoy G, Lamark T, Brech A, Outzen H, Perander M, Overvatn A, et al. p62/SQSTM1 forms protein aggregates degraded by autophagy and has a protective effect on huntingtin-induced cell death. The Journal of cell biology. 2005;171:603-14.

Chen Q, Cai ZK, Chen YB, Gu M, Zheng DC, Zhou J, et al. Poly $r(C)$ binding protein-1 is central to 
maintenance of cancer stem cells in prostate cancer cells. Cellular physiology and biochemistry: international journal of experimental cellular physiology, biochemistry, and pharmacology. 2015;35:1052-61.

Degenhardt K, Mathew R, Beaudoin B, Bray K, Anderson D, Chen G, et al. Autophagy promotes tumor cell survival and restricts necrosis, inflammation, and tumorigenesis. Cancer cell. 2006;10:51-64.

Deretic V. Autophagosome and phagosome. Methods in molecular biology. 2008;445:1-10.

Fulda S, Debatin KM. Extrinsic versus intrinsic apoptosis pathways in anticancer chemotherapy. Oncogene. 2006;25:4798-811.

Galluzzi L, Pietrocola F, Bravo-San Pedro JM, Amaravadi RK, Baehrecke EH, Cecconi F, et al. Autophagy in malignant transformation and cancer progression. The EMBO journal. 2015;34:856-80.

Giles KM, Daly JM, Beveridge DJ, Thomson AM, Voon DC, Furneaux HM, et al. The 3'-untranslated region of p21WAF1 mRNA is a composite cis-acting sequence bound by RNA-binding proteins from breast cancer cells, including HuR and poly(C)-binding protein. The Journal of biological chemistry. 2003;278:2937-46.

Huang S, Okamoto K, Yu C, Sinicrope FA. p62/sequestosome-1 up-regulation promotes ABT-263-induced caspase-8 aggregation/activation on the autophagosome. The Journal of biological chemistry. 2013;288:33654-66.

Huang YH, Al-Aidaroos AQ, Yuen HF, Zhang SD, Shen HM, Rozycka E, et al. A role of autophagy in PTP4A3-driven cancer progression. Autophagy. 2014;10:1787-800.

Janku F, McConkey DJ, Hong DS, Kurzrock R. Autophagy as a target for anticancer therapy. Nature reviews Clinical oncology. 2011;8:528-39.

Kim JH, Hong SK, Wu PK, Richards AL, Jackson WT, Park JI. Raf/MEK/ERK can regulate cellular levels of LC3B and SQSTM1/p62 at expression levels. Experimental cell research. 2014;327:340-52.

Klionsky DJ, Abdalla FC, Abeliovich H, Abraham RT, Acevedo-Arozena A, Adeli K, et al. Guidelines for the use and interpretation of assays for monitoring autophagy. Autophagy. 2012;8:445-544.

Kruidering M, Evan GI. Caspase-8 in apoptosis: the beginning of "the end"? IUBMB life. 2000;50:85-90.

Levine B. Cell biology: autophagy and cancer. Nature. 2007;446:745-7.

Li J, Yuan J. Caspases in apoptosis and beyond. Oncogene. 2008;27:6194-206.

Liu Y, Gai L, Liu J, Cui Y, Zhang Y, Feng J. Expression of poly(C)-binding protein 1 (PCBP1) in NSCLC as a negative regulator of EMT and its clinical value. International journal of clinical and experimental pathology. 2015;8:7165-72.

Lu Z, Baquero MT, Yang H, Yang M, Reger AS, Kim C, et al. DIRAS3 regulates the autophagosome initiation complex in dormant ovarian cancer cells. Autophagy. 2014;10:1071-92.

Maiuri MC, Criollo A, Kroemer G. Crosstalk between apoptosis and autophagy within the Beclin 1 interactome. The EMBO journal. 2010;29:515-6.

Malik AK, Flock KE, Godavarthi CL, Loh HH, Ko JL. Molecular basis underlying the poly C binding protein 1 as a regulator of the proximal promoter of mouse mu-opioid receptor gene. Brain research. 2006;1112:33-45. 
Mizushima N. Autophagy: process and function. Genes \& development. 2007;21:2861-73.

Moscat J, Diaz-Meco MT. p62 at the crossroads of autophagy, apoptosis, and cancer. Cell. 2009;137:1001-4.

Nishinakamura H, Minoda Y, Saeki K, Koga K, Takaesu G, Onodera M, et al. An RNA-binding protein alphaCP-1 is involved in the STAT3-mediated suppression of NF-kappaB transcriptional activity. International immunology. 2007;19:609-19.

Pankiv S, Clausen TH, Lamark T, Brech A, Bruun JA, Outzen H, et al. p62/SQSTM1 binds directly to Atg8/LC3 to facilitate degradation of ubiquitinated protein aggregates by autophagy. J Biol Chem. 2007;282:24131-45.

Ravikumar B, Sarkar S, Davies JE, Futter M, Garcia-Arencibia M, Green-Thompson ZW, et al. Regulation of mammalian autophagy in physiology and pathophysiology. Physiological reviews. 2010;90:1383-435.

Rivera-Gines A, Cook RJ, Loh HH, Ko JL. Interplay of Sps and poly(C) binding protein 1 on the mu-opioid receptor gene expression. Biochemical and biophysical research communications. 2006;345:530-7.

Sun K, Deng W, Zhang S, Cai N, Jiao S, Song J, et al. Paradoxical roles of autophagy in different stages of tumorigenesis: protector for normal or cancer cells. Cell \& bioscience. 2013;3:35.

Tang Y, Li M, Wang YL, Threadgill MD, Xiao M, Mou CF, et al. ART1 promotes starvation-induced autophagy: a possible protective role in the development of colon carcinoma. American journal of cancer research. 2015;5:498-513.

Valente G, Morani F, Nicotra G, Fusco N, Peracchio C, Titone R, et al. Expression and clinical significance of the autophagy proteins BECLIN 1 and LC3 in ovarian cancer. BioMed research international. 2014;2014:462658.

Wang H, Vardy LA, Tan CP, Loo JM, Guo K, Li J, et al. PCBP1 suppresses the translation of metastasis-associated PRL-3 phosphatase. Cancer cell. 2010;18:52-62.

Wu H, Che X, Zheng Q, Wu A, Pan K, Shao A, et al. Caspases: a molecular switch node in the crosstalk between autophagy and apoptosis. Int J Biol Sci. 2014;10:1072-83.

Young MM, Takahashi Y, Khan O, Park S, Hori T, Yun J, et al. Autophagosomal membrane serves as platform for intracellular death-inducing signaling complex (iDISC)-mediated caspase-8 activation and apoptosis. The Journal of biological chemistry. 2012;287:12455-68.

Zhang T, Huang XH, Dong L, Hu D, Ge C, Zhan YQ, et al. PCBP-1 regulates alternative splicing of the CD44 gene and inhibits invasion in human hepatoma cell line HepG2 cells. Molecular cancer. 2010;9:72.

Zhao Y, Chen S, Gou WF, Xiao LJ, Takano Y, Zheng HC. Aberrant Beclin 1 expression is closely linked to carcinogenesis, differentiation, progression, and prognosis of ovarian epithelial carcinoma. Tumour biology : the journal of the International Society for Oncodevelopmental Biology and Medicine. 2014;35:1955-64. 


\section{Figure legends}

Figure 1. PCBP1 overeepression represses tumor cell proliferation under serum-free conditions.

(A) Western blots of stable overexpression of GFP-PCBP1 in A2780 and DLD-1 cells with PCBP1 antibody. GAPDH is used as loading control.

(B) and (C) CCK8 cell proliferation analyses of A2780 (B) and DLD-1 (C) cells with exogenous PCBP1 in serum-free starvation in the absence or presence of autophagic inhibitor CQ at $50 \mu \mathrm{M}$. Data presented are mean \pm SD. NS: No Significance; $* * * P<0.001$, $n=3$.

(D) Western blots of LC3B I and II in A2780 and DLD-1 cells with stable overexpression of GFP-PCBP1 and their parental GFP counterparts. Cells were starved in EBBS media for the indicated time, compared to the normal culture medium (Control). GAPDH is used as loading control. Relative expression of LC3B I and II to GAPDH are shown under each lane. Long and Short represent the longer and shorter time of film exposures.

Figure 2. PCBP1 Overexpression represses starvation-induced autophagic flux.

(A) Western blots of LC3B I, II and p62 in A2780 and DLD-1cells with GFP-PCBP1 (PCBP1) and the GFP-expressing cells. Cells were cultured in normal medium, or EBSS medium with or without CQ for the indicated time and analyzed. Protein bands' intensity ratio of LC3B II/GAPDH as autopahgic flux was quantified and normalized, which are shown under each lane. Long and Short represent the longer and shorter time of film 
exposures.

(B) and (C) Immunofluorescence staining of LC3B puncta (in Red) in A2780 (B) and

DLD-1 cells (C). GFP-PCBP1 cells are in green, and their control cells are counterstained with DAPI in blue. Cells were treated with CQ combined EBSS starvation at indicated time and stained with anti-LC3B antibody. White arrows point LC3B puncta in control cells, and the red in PCBP1 overexpressing cells. Bars equal to $50 \mu \mathrm{m}$.

(D) Quantification of LC3B puncta intensity (number) in B and C. At least 25 cells from 5 different viewing fields were counted as described in materials and method and presented as a histogram (mean $\pm \mathrm{SD}), * P<0.05 ; * * P<0.01$; *** $P<0.001, \mathrm{n}=5$.

\section{Figure 3. PCBP1 Knockdown promotes starvation-induced autophagic flux.}

(A) Western blots of endogenous PCBP1 in A2780, HCT-116 and CHO cells with PCBP1 antibody. Four shRNAs against PCBP1 were transfected into cells to establish the indicated cell pools (KD), and the empty vector was used for the control cells (GFP). GAPDH was used as loading control.

(B) Western blots of LC3B I, II and p62 in A2780 and HCT-116 cells with PCBP1 knockdown (KD) as well as the control cells (GFP), shown in A. Cells were cultured in normal medium, or EBSS medium with or without CQ for the indicated time and analyzed. Protein bands' intensity ratio of p62 and LC3B II to GAPDH were quantified and normalized to be shown under each lane, respectively.

(C) and (D) Immunofluorescence staining of LC3B puncta (in Red) in CHO (C) and HCT-116 cells (D). PCBP1 KD HCT-116 cells are in green, and their control cells are counterstained with DAPI in blue. Cells were treated with CQ combined EBSS starvation 
for 2 hours and fixed for IF with anti-LC3B antibody. White arrows point LC3B puncta in PCBP1 KD cells, and the red in control cells. Bars equal to $50 \mu \mathrm{m}$.

\section{Figure 4. PCBP1 attenuates LC3B mRNA stability.}

(A). Immunoblot of ectopic GFP-PCBP1 and endogenous PCBP1 protein expression in the indicated cells. PCBP1 is silenced by its two specific shRNAs.

(B) Semi-quantitative RT-PCR analysis of LC3B mRNA stability in A2780 cells overexpressing PCBP1 or with endogenous PCBP1 knockdown (KD), compared to GFP controls upon $50 \mu \mathrm{M}$ Actinomycin D (Act D) treatment to terminate the novel mRNA transcription at the indicated time points. GAPDH were used as internal control, and relative LC3B mRNA levels are normalized to those of GAPDH, and shown under each lane.

(C) Schematic curve of LC3B mRNA stability along with Act D treatment period in B.

(D) Semi-quantitative RT-PCR analysis of LC3B mRNA stability in DLD-1 cells overexpressing PCBP1, compared to GFP controls as in B.

(E) Schematic curve of LC3B mRNA stability along with Act D treatment period in D.

(F) Immunoblot of LC3B protein levels in the cells upon CQ treatment at the indicated time points. Results are representative of at least three independent experiments. The relative LC3B II protein level is normalized to GAPDH protein level and shown under each lane.

Figure 5. PCBP1 overexpression enhances tumor cell death under nutrient 


\section{deprivation.}

(A) Cell death analyses of A2780 and DLD-1 with PCBP1 overexpression, compared with the parental GFP cells. Cells were seeded in 6-well and cultured overnight, then treated by EBSS with or without CQ for $36 \mathrm{~h}$, and photographed.

(B) Statistical analyses of cell death shown in A. At least 75 cells from 6 distinct viewing fields were counted and presented as a histogram (mean $\pm \mathrm{SD}$ ), NS: No Significance; $* P<$ $0.05 ; * * P<0.01 ; * * * P<0.001, \mathrm{n}=6$.

(C) and (D) Flow cytometric analyses of apoptotic cells in A2780 cells (C) and DLD-1 cells (D) with PCBP1 overexpression (GFP-PCBP1), compared with their control cells (GFP). The detailed procedure was presented in the Materials and Methods. Cells were cultured in normal medium (control) or EBBS with or without autophagic inhibitor treatment. Overall percentages of the apoptotic cells are shown as the sum of Annexin-V positive cells.

Figure 6. PCBP1 overexpression enhances the activation of caspases under nutrient deprivation.

(A). Western blots of the indicated proteins with their specific antibodies in A2780 and DLD-1 cells. Cells with PCBP1 overexpression (PCBP1) and their control cells (GFP) were treated with EBSS or EBSS combined with CQ at indicated time before lyses. GAPDH was used as loading control. Arrows indicate the molecular weight of each protein. Cleaved forms of proteins shown as c-protein name.

(B). Western blots of the indicated proteins with their specific antibodies in HCT-116 cells 
with endogenous PCBP1 knockdown, compared to their parental GFP controls as in A. 
A

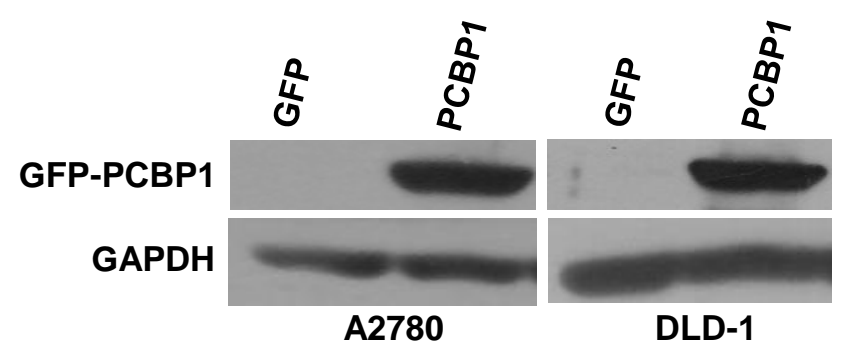

B

C
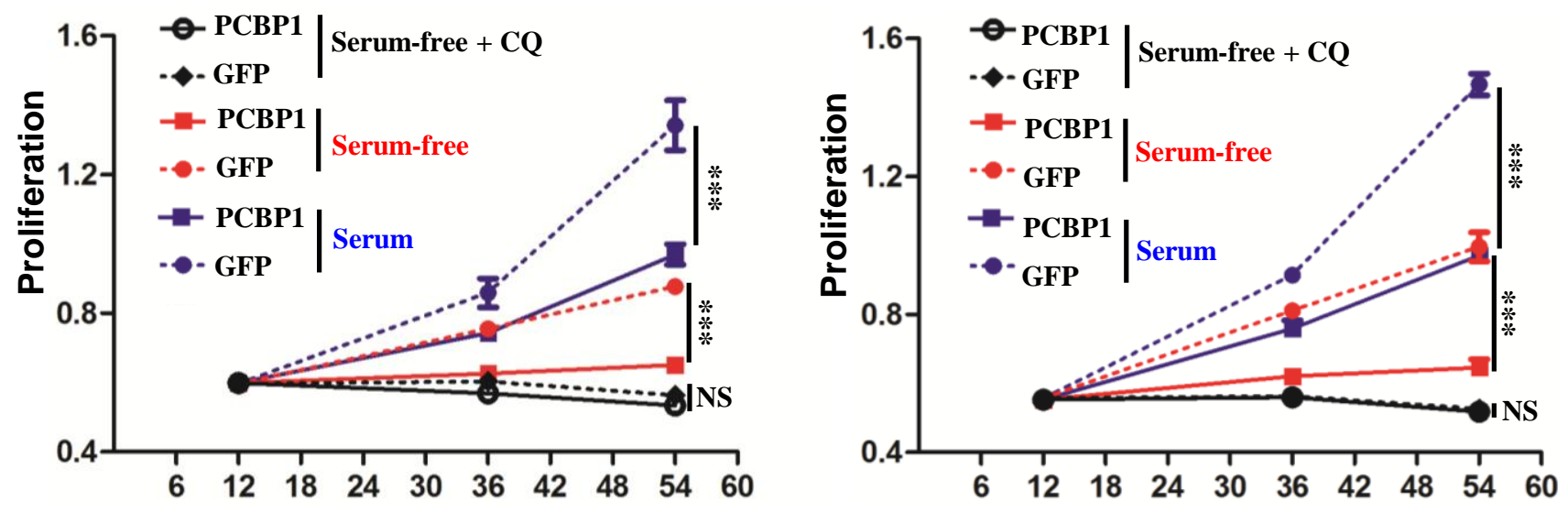

D

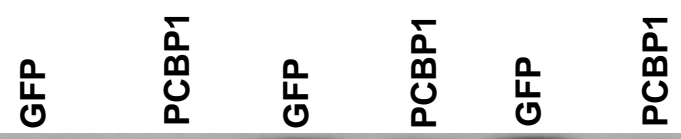

$\begin{array}{ll}\frac{n}{0} & 0 \\ \frac{0}{0} & 0\end{array}$

㱏

品 品

足
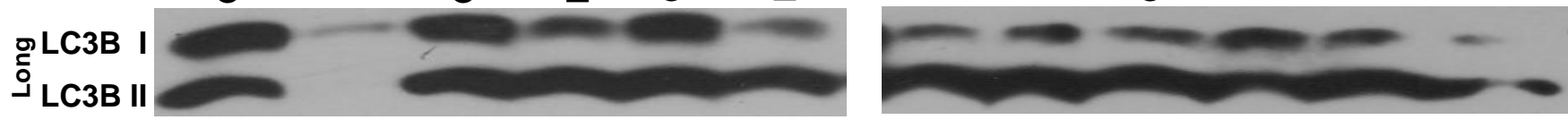

는 $3 B$ ।
๘ LC3B II

LC3BI+II/GAPDH 1.27

$0.12 \quad 0.92$

0.76

$\begin{array}{ll}0.99 & 0.27\end{array}$

GFP-PCBP1

GAPDH

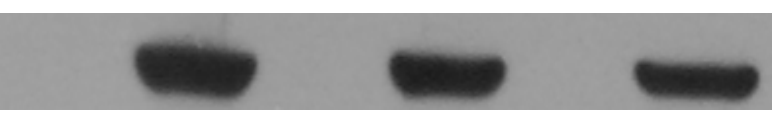

$3.11 \quad 2.52$

2.75

2.41

2.56

0.72
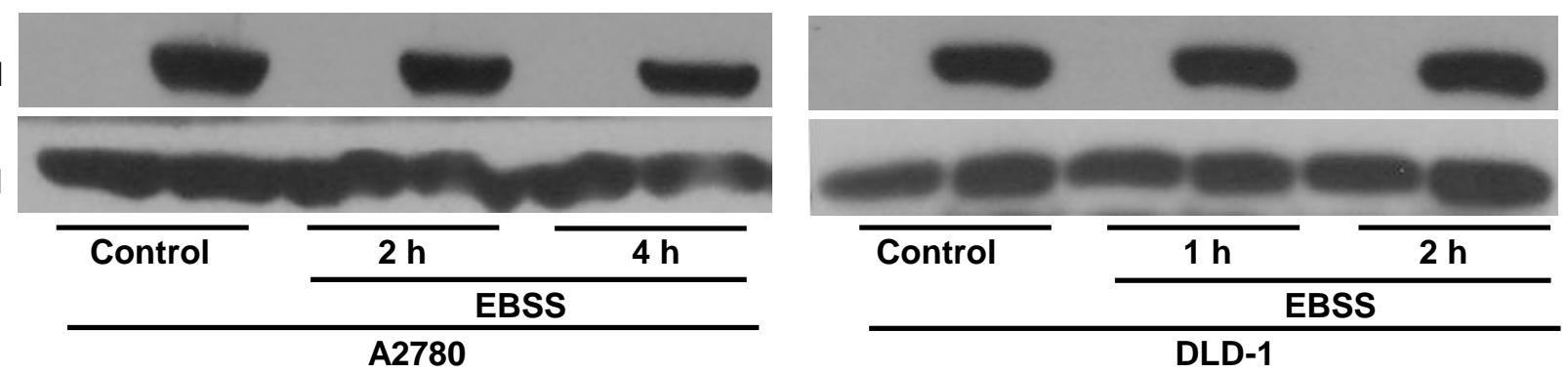
A

D

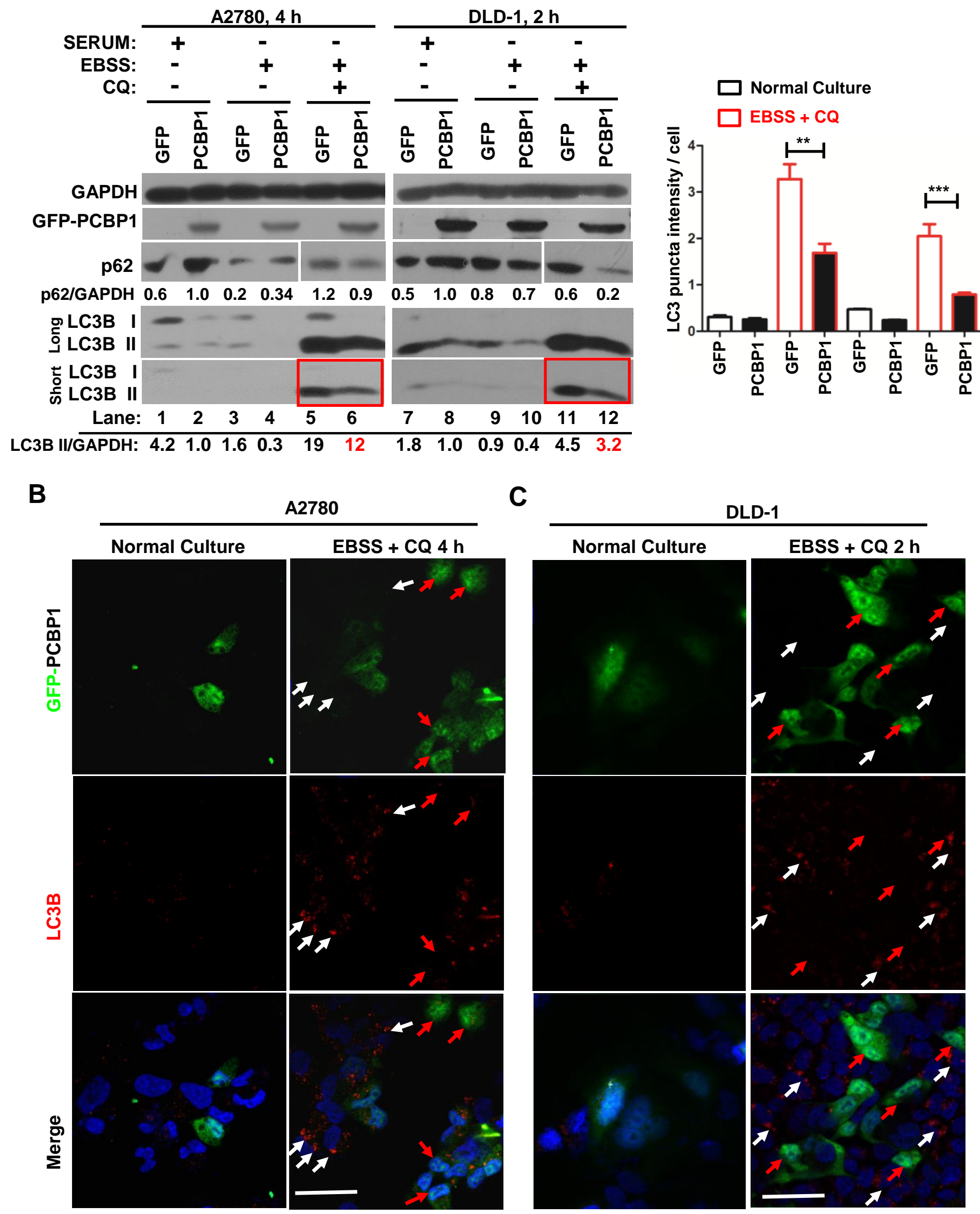

Figure 2 
A

B
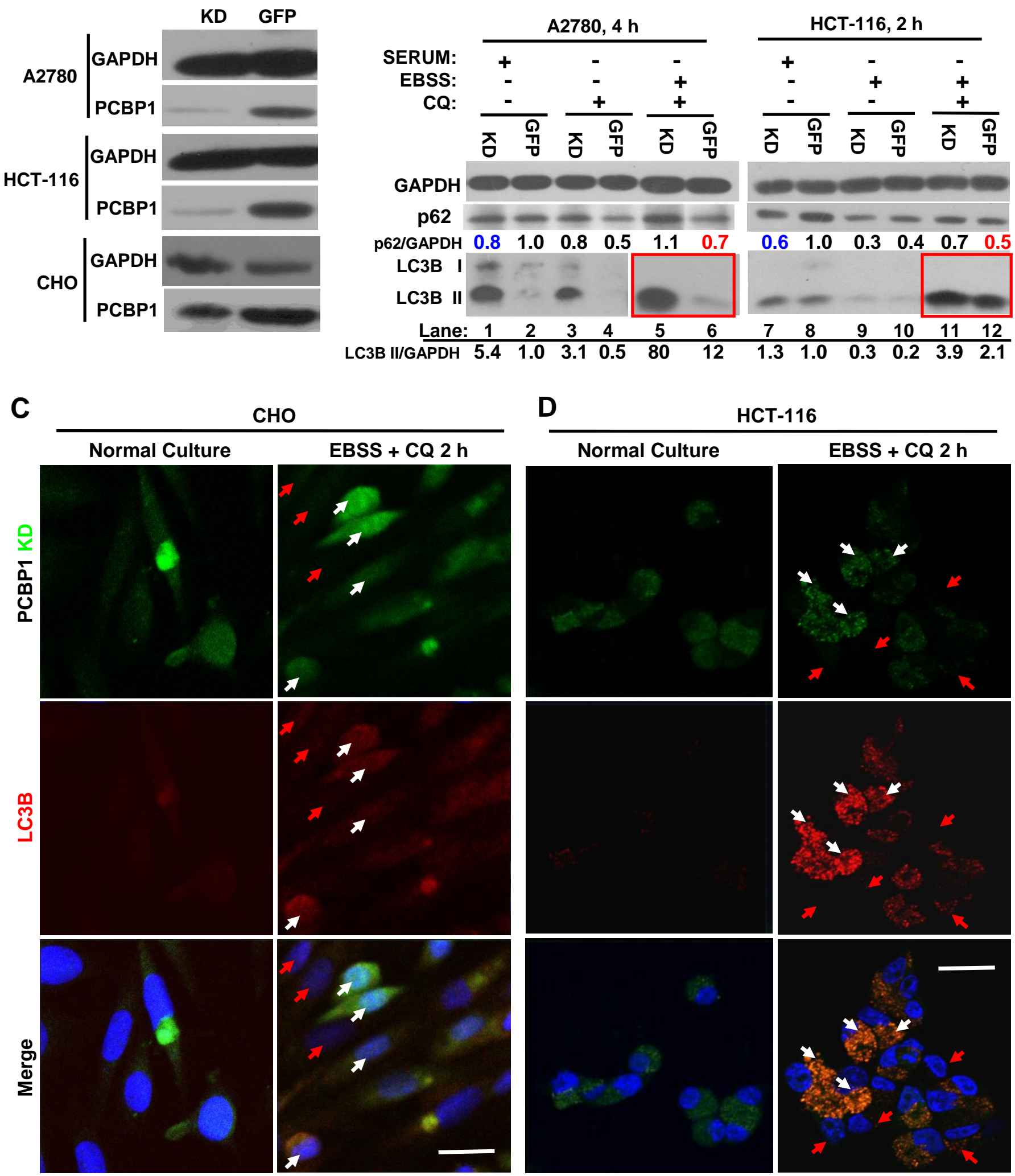

D

HCT-116

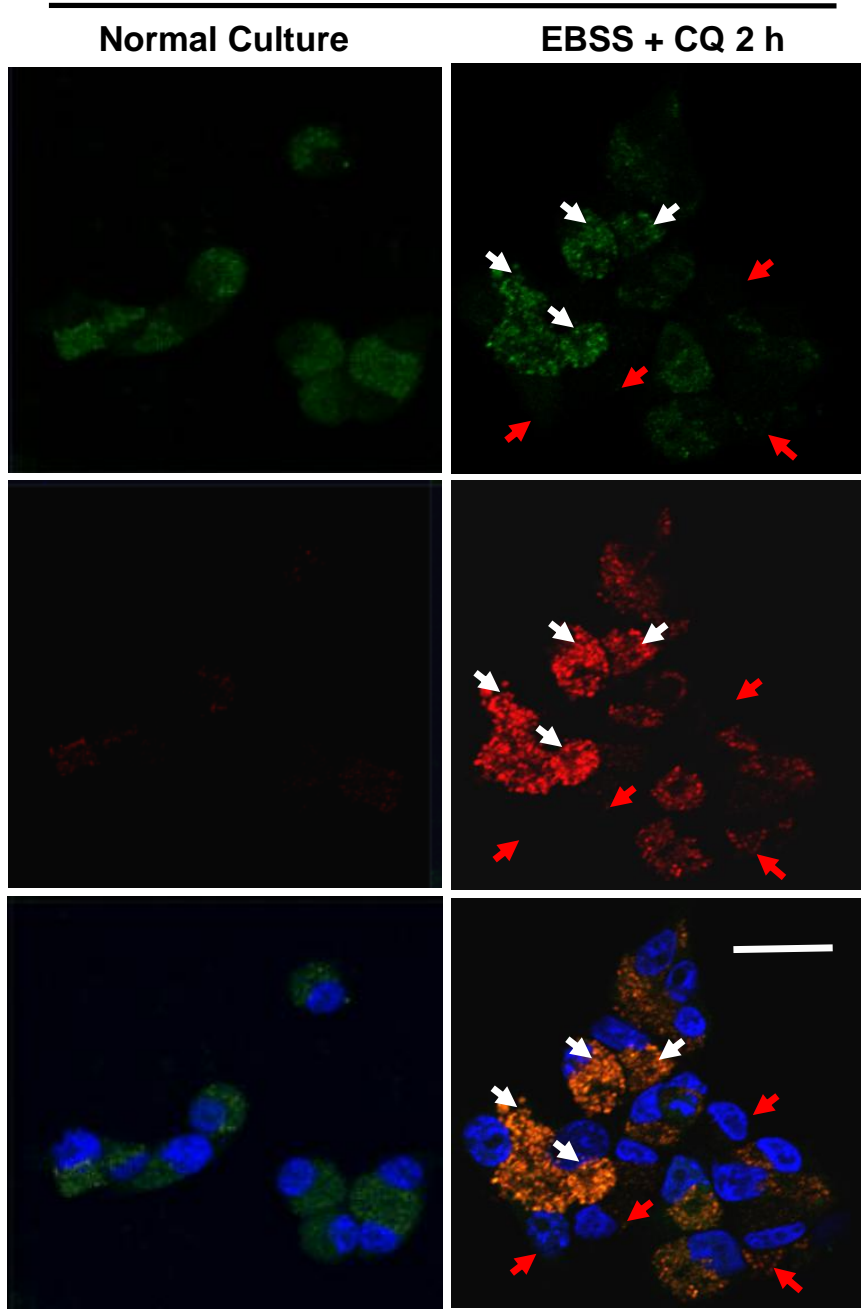

Figure 3 
A

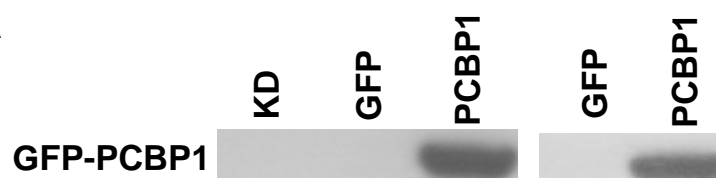

Endo-PCBP1

GAPDH

$\begin{array}{ll}\text { A2780 DLD-1 } & \end{array}$

B

B C
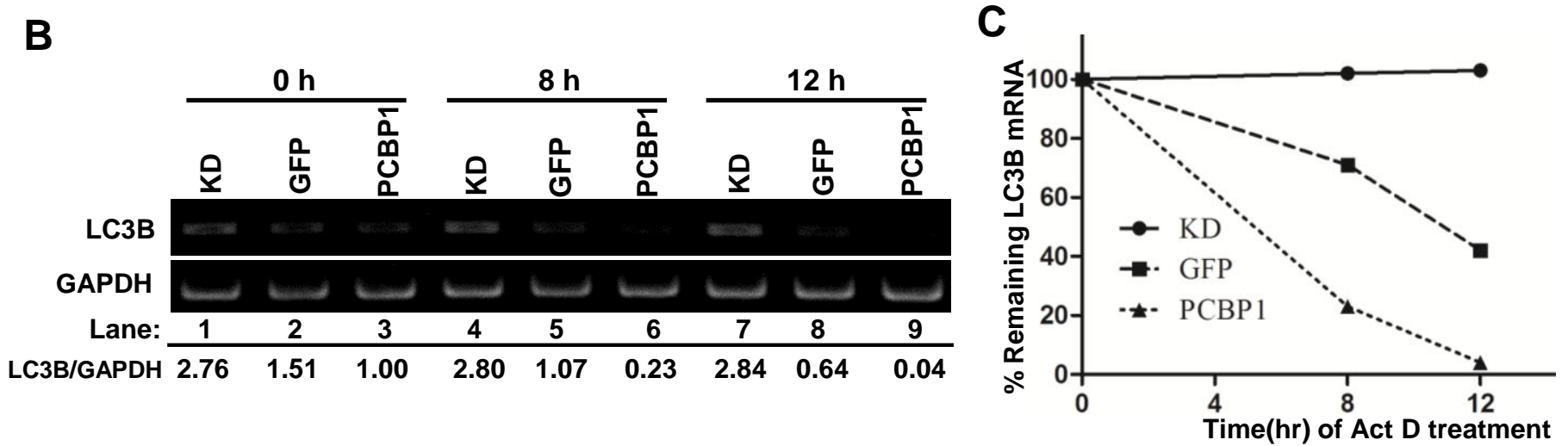

D

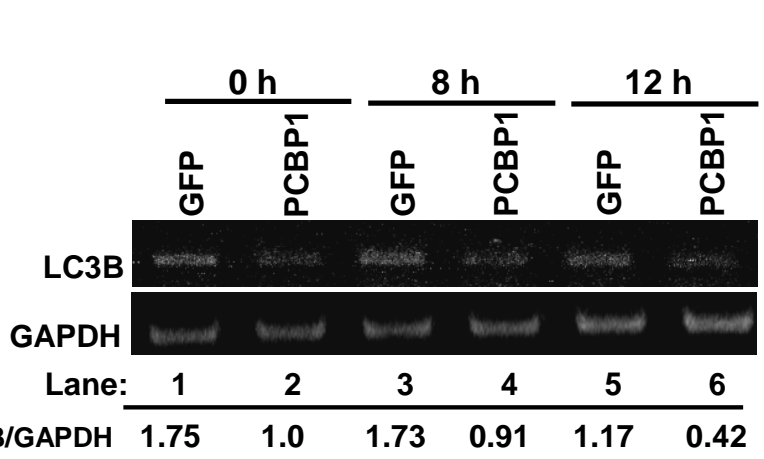

E

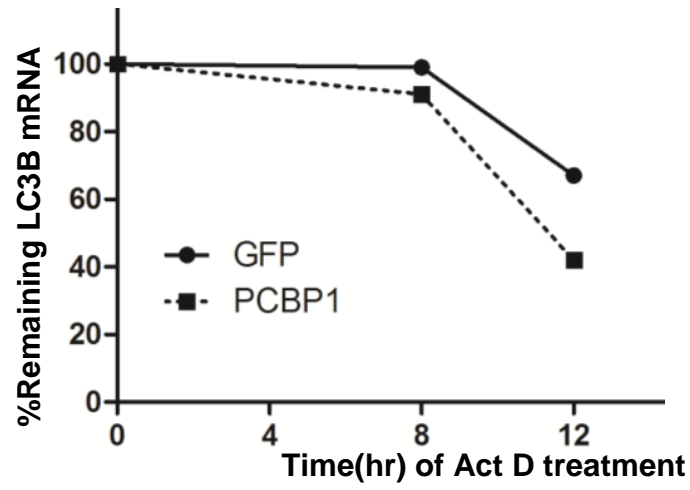

$\mathbf{F}$

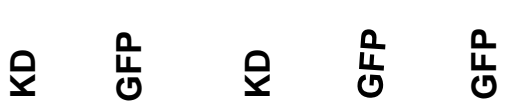

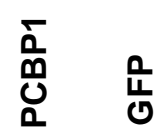

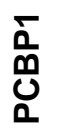

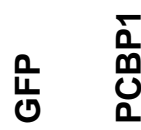
空
空

LC3B I

LC3B II

LC3B II/GAPDH:

$\begin{array}{llll}0.3 & 0.1 & 1.7 & 1.0\end{array}$

$\begin{array}{llll}0.2 & -- & 1.0 & 0.6\end{array}$

$\begin{array}{llll}0.2 & 0.06 & 1.0 & 0.6\end{array}$

PCBP1

GAPDH
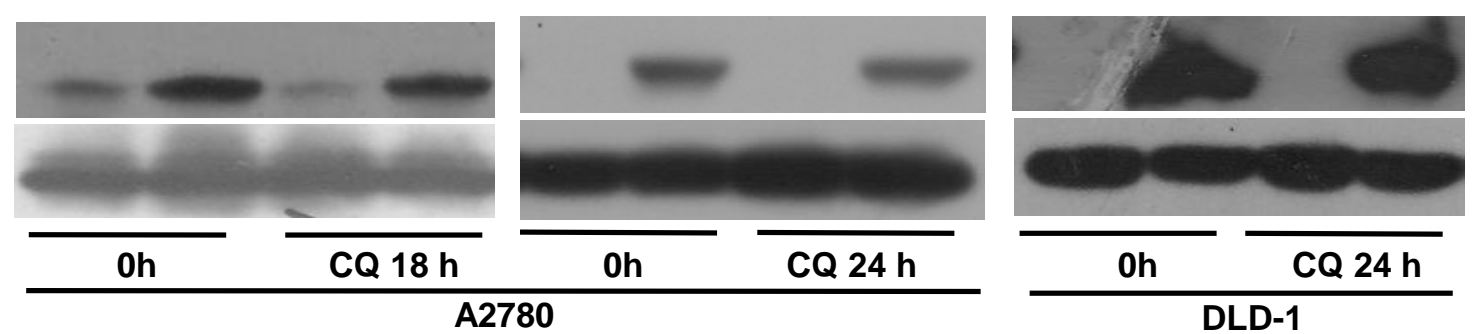

Figure 4 
A

DLD-1
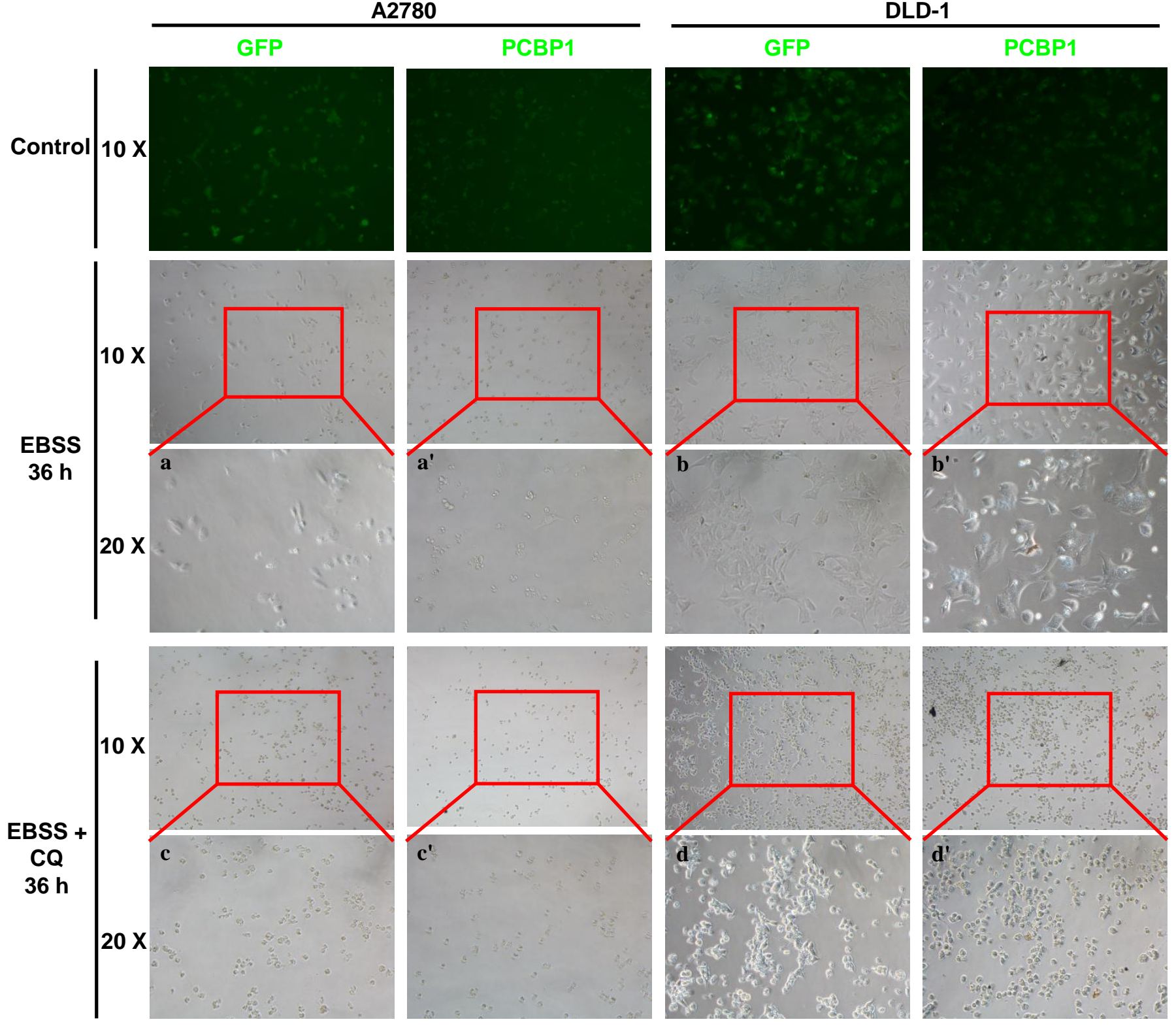

B

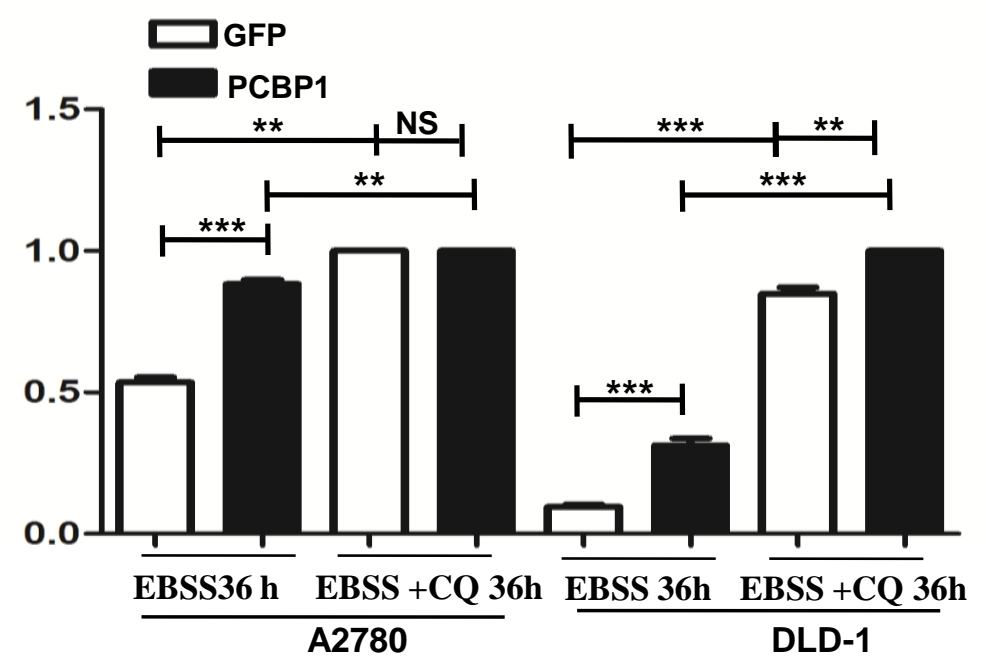

Figure 5 
C
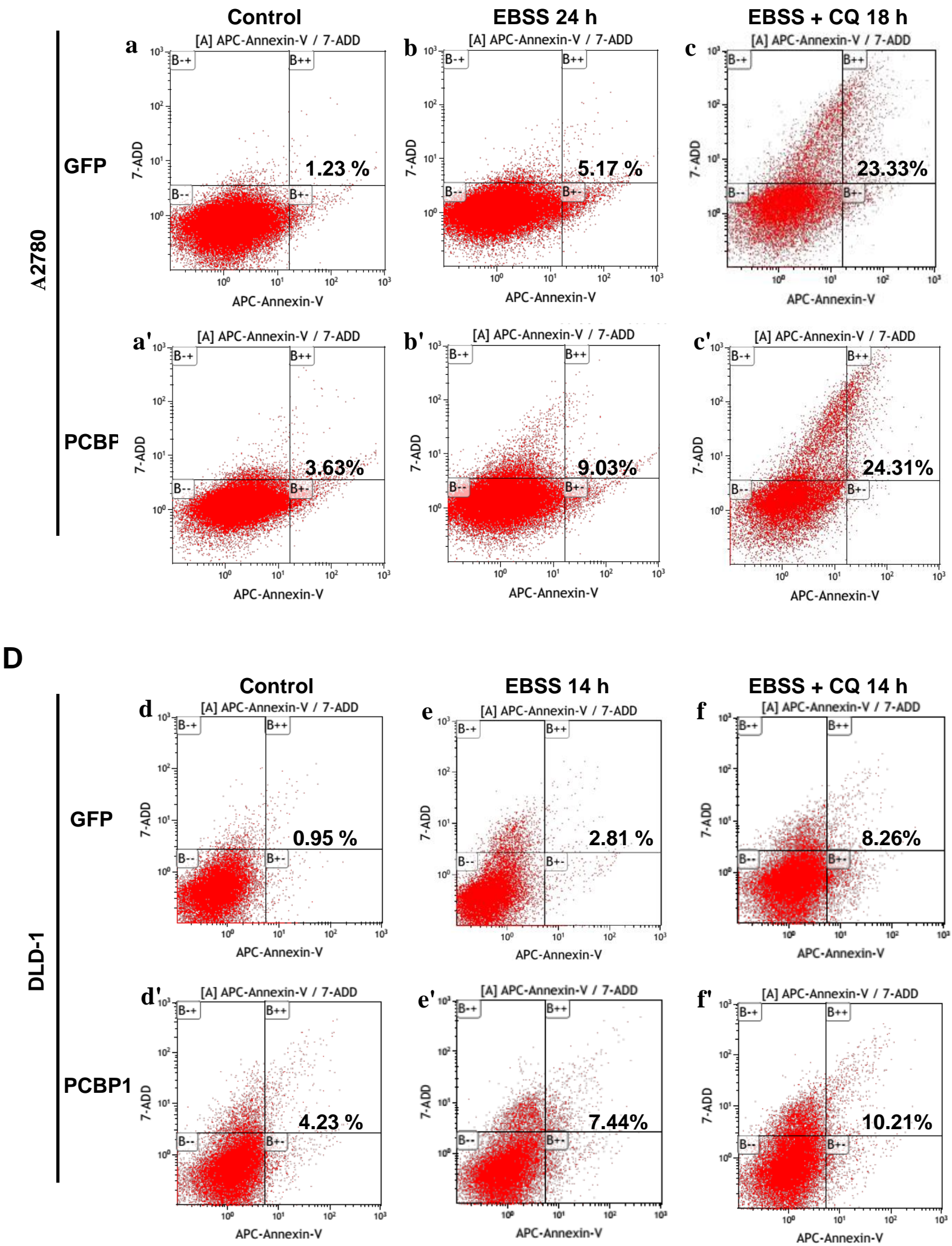

Figure 5 
A

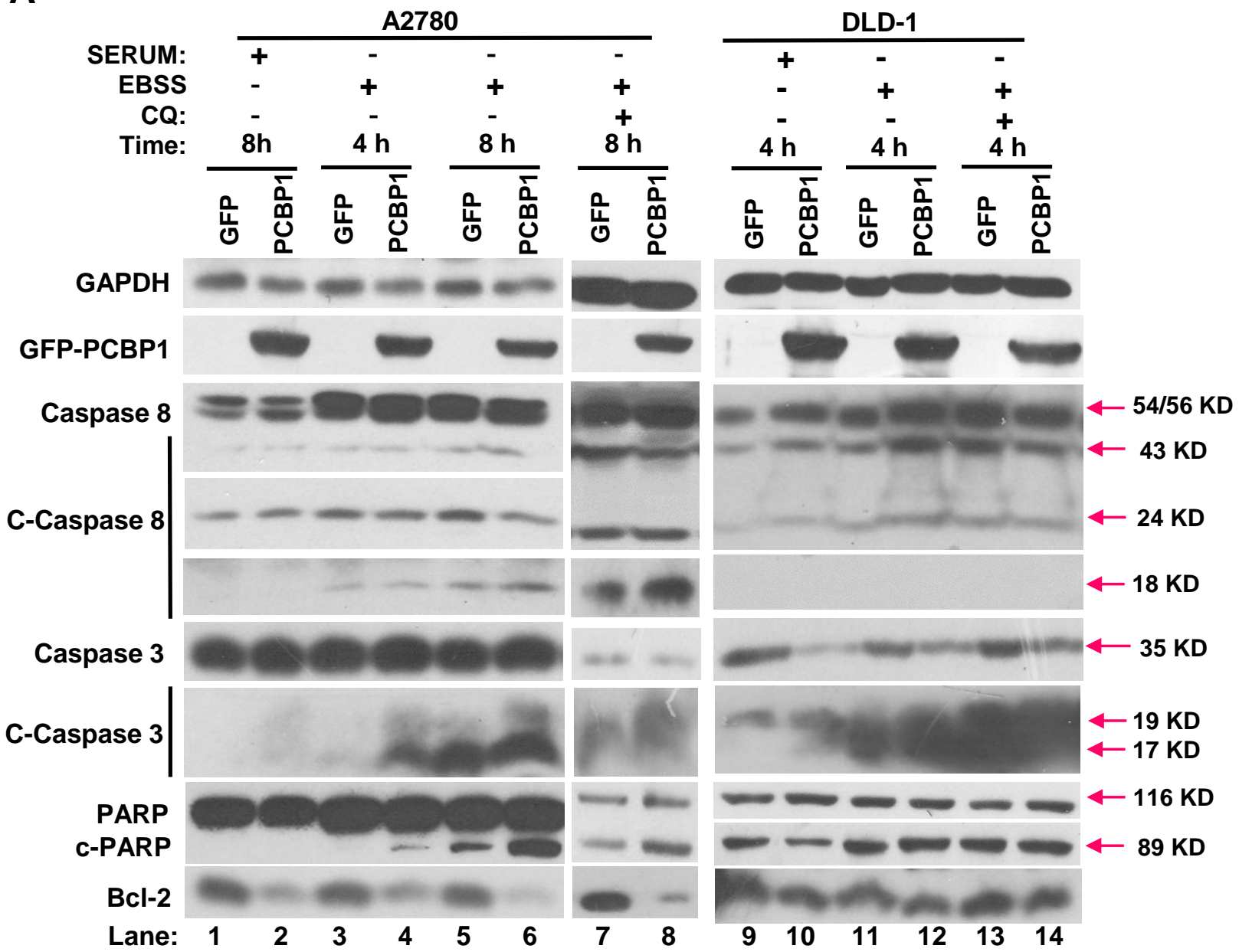

B

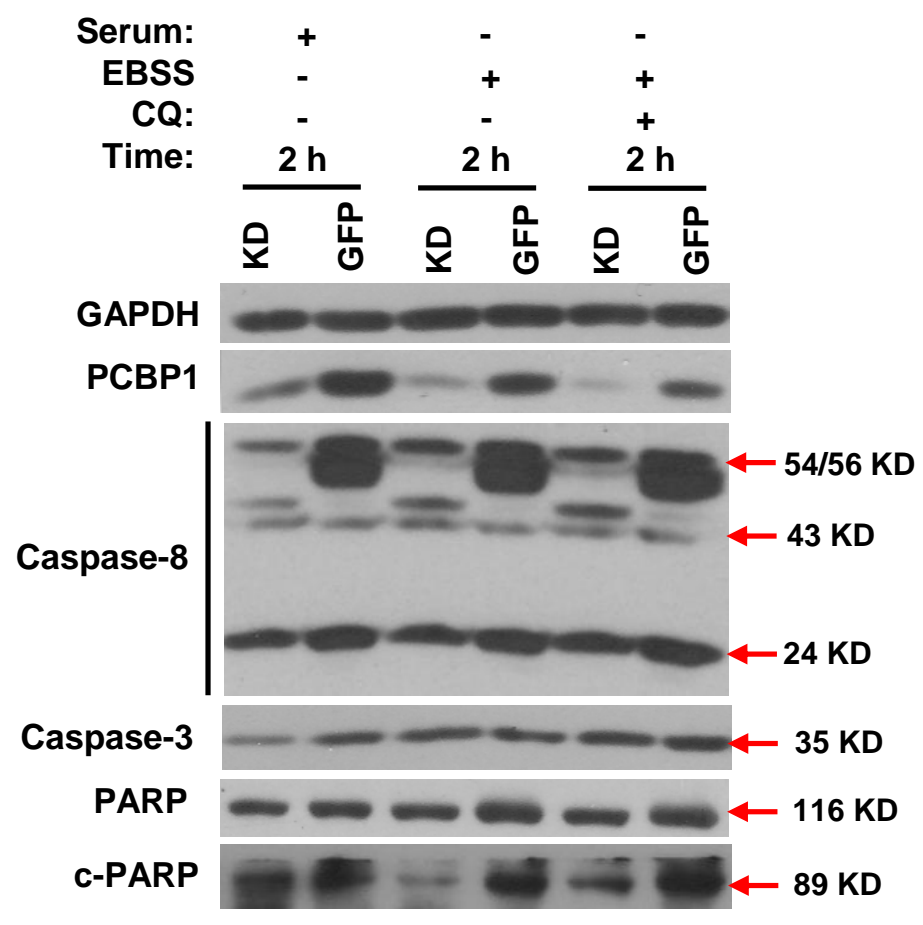

Figure 6 